\title{
Past Participles in Multiword Units in Croatian
}

Key words: adjectivisation, collocations, Croatian dictionaries, multiword units, past participles

\section{Introduction: The Past Participle in a Theoretical and Lexicographic} Framework $^{1}$

Croatian past participles ending with - $n$ (and - an, - en as allomorphs) and - $t$, and Croatian true (descriptive, possessive, and material ${ }^{2}$ ) adjectives have similar features as concerns their syntactic organisation. With the modal verb biti ("to be"), they express the passive voice of a predicate: Cuijet je ubran - "The flower has been picked" (ubran is the past participle form of the verb ubrati - "to pick") / Cuijet je lijep - "The flower is beautiful" (lijep is the true adjective). Like true adjectives (ZNIKA, 2005), past participles, when used in combination with nouns, can be used attributively and assume the grammatical features of nominal words (SEIFART, 2010) such as gender, number, and case (e.g. in nadahnut govor"inspired speech" - nadahnut is the past participle of the verb nadahnuti - "to inspire"). Aside from these similarities between past participles and true adjectives, related works (ZNIKA, 2005: 434) point to differences in grammatical meaning, which is based on the ability of the past participles to express the passive voice of the transitive verbs which they belong to paradigmatically, while true adjectives express the static characteristic of a noun. Also, particular explanations (ZNIKA, 2005: 437) point to the existence of two homonymous forms: e.g. the forms dimljen ("smoked"), mljeven ("minced"), and tučen ("beaten") can be qualified either as the past participle of the verbs dimiti ("to smoke"), mljeti ("to mince"), and tući ("to beat"), respectively, or as true adjectives. The homonymy criterion is useful in the inclusion of adjectives (both past participles and true adjectives) in dictionary entries. I am also aware that this problem would be much clearer if other

*e-mail: gblagus@ihjj.hr

1 Acknowledgements: The research reported in this paper is supported by the Project MREŽNIK Hruatski mrežni rječnik / The Croatian Web Dictionary Project, IP-2016-06-2141 (http://ihjj.hr/projekt /hruatski-mrezni-rjecnik-mreznik/70/), Hruatska zaklada za znanost / Croatian Science Foundation (https://epp.hrzz.hr/default.aspu).

2 Material adjectives (e.g. Engl. wooden, glass, golden) describe the materials that a noun consists of. 
Slavic languages (other South Slavic languages, Czech, Polish, Russian) were included in the analysis, especially if we consider $-n /-t$ participles to be a ubiquitous phenomenon in Slavic languages (SMITH, 1995; MARvin, 2003; KIBORT, 2012). Such comparative analysis will be the subject of future research.

Traditionally, past participles are listed in Croatian dictionaries most often as a morphological form under an infinitive as a canonical form of verbal entries. They are rarely represented as adjectival entries (e.g. dimljen - "smoked," usoljen - "salted, salt-cured").

According to the aforementioned theoretical and lexicographic assumptions, past participles and true adjectives have many features in common. The accent shall be placed on adjectival features of the past participle; we will, therefore, consider those cases in which the past participles show grammatical and semantic features of true adjectives (case, gender, number, usage and meaning). Also, two other features are important to consider when we observe past participles as true adjectives: (1) past participles denote gradable concepts through comparative and superlative forms, as is noted in Croatian (ZNIKA, 2005: 430-431) and in Polish (КівоRт, 2012: 331); and (2) at the semantic level, past participles lose their features of passive predicative forms that modify the subject as a patient and, instead, behave as descriptive adjectives agreeing with the head nouns (KIBORT, 2012: 331). ${ }^{3}$ Grammatically, past participles are formed from transitive verbs in Croatian - they are object-oriented forms (CetnARowsKA, 2000: 47, according to: Nedjalkov, Jaxontov, 1988: 6) which, when used as adjectives in a nominal phrase (NP), determine the object noun (mljeti meso - "to mince meat" / mljeveno meso - "minced meat").

Given this theoretical framework, the further discussion will be based on the analysis of past participles in various multiword units (MWUs) in Croatian. The main goal of this research is to show whether the level of lesicalisation, i.e. the adjectivisation of past participles, can be determined by their collocational potential on the basis of their cooccurrence with other words (nouns). This approach is based on the explanation provided in IVIR (1992/1993: 185-186), which separates the prototypical, core meaning of words that changes in collocational relations, leading to the conclusion that the prototypical meaning of some words is only the initial, semantic potential they realise in various specified meanings depending on their collocational relationship with other words.

An overview of the usage of past participles in multiword units (MWUs) shall be shown on the examples taken from the Croatian Collocation Database (CCD). ${ }^{4}$ In short, the CCD is primarily based on the traditional lexicographic and lesicological settings of multiword lerical units (Benson et al., 1997; Mel'čuk, 2001 [1998]; Sag et al., 2002; Blagus Bartolec, 2014). ${ }^{5}$ The data sources for CCD include: Croatian daily, weekly, and monthly newspapers; various online sources; contemporary Croatian lesicographical manuals (dictionaries,

${ }^{3}$ E.g. in meso je mljeveno u mašini ("meat was minced in machine") meat is a patient of passive predicative form was minced while in minced meat past participle minced has a descriptive adjectival meaning.

${ }^{4}$ For more on the CCD, see: http://ihjj.hr/kolokacije/. All past participles listed in this paper have been included in the CCD.

${ }^{5}$ The CCD will be a useful source to be included in other more advanced MWU sources (both Croatian and international) for the development of tools for extracting MWUs on the basis of their semantic and lexical features ( $\mathrm{RAMISCH}, 2015)$. 
lexicons, encyclopedias); Narodne novine online (the official newspaper of the Republic of Croatia); recent linguistic journals with articles on the topics of word combinations in Croatian; and Croatian web corpus - hrWaC (http://nlp.ffzg.hr/resources/corpora/hrwac/).

Generally, the concept behind the CCD is to create a database with the most common Croatian multiword units by defining their semantic types and usage contert: idioms (e.g. ustati na lijevu nogu, literally "to get up on one's left leg," Engl. "to get up on the wrong side of the bed"), multiword terms that are specific to a particular scientific or professional field (e.g. anodna korozija - "anode corrosion," običajno pravo - "customary law"), proverbs (e.g. u laži su kratke noge, literally "lies have short legs," Engl. "lies don't travel far"), collocations (word combinations with more restricted or specific meanings, e.g. vitke noge "slender legs"), free combinations (combinations with freedom of selection and freedom of combination, e.g. prekrižiti noge - "to cross (one's) legs").

All of the aforementioned types of lexical units as parts of the CCD frequently contain past participles (e.g. odrezak pečen na žaru, literally "a steak baked on ember," Engl. "grilled steak," promet je pojačan, literally "traffic is intensified," Engl. "traffic is heavy," zrak je onečišćen - "the air is polluted," zuijezda je rođena - "a star is born," električno nabijena čestica - "electrically charged particle," stečena prava i dužnosti - "attained rights and duties") which are expanded in various styles of Croatian (journalism, administration and legislation, professional and scientific literature, etc.). The CCD contains several columns, the first of which provides the entry that is a component of a particular unit. The entries appear in their canonical form (infinitive for verbs, nominative for nouns, masculine gender for adjectives and pronouns). In the case of past participles that are part of various MWUs (i.e. idioms or multiword terms), it is occasionally impossible to unambiguously determine whether their canonical form is that of a verb or of an adjective. For the needs of this research, 393 past participles ending in - $n$ (and -an, - en as allomorphs) and - $t$ were extracted from the CCD. The status of these forms was checked in four modern Croatian dictionaries: Rječnik hruatskoga jezika (Dictionary of the Croatian Language, hereinafter: RHJ), Školski rječnik hruatskoga jezika (Scholastic Croatian Dictionary, hereinafter: ŠKRJ), Veliki rječnik hruatskoga standardnog jezika (Large Dictionary of the Croatian Language, hereinafter: VRH), and Hrvatski jezični portal (Croatian Language Portal, hereinafter: HJP). Of the past participles examined, 218 are listed in one or more of the mentioned dictionaries as independent adjectival entries, and can thus be considered true adjectives. The other 175 past participles in existing Croatian dictionaries are not listed as independent adjectival entries, and are rather listed under verbal entries. According to the attestations in the CCD, these past participles also act as true adjectives in various multiword units. Due to the limited length of the article, I have not included all of these forms here, but a large number of екаmples will be listed in the following sections.

I examine past participles that are not listed as independent entries in any dictionary, but which appear in usage as adjectival collocations in multiword units. On the basis of data from these four dictionaries and a comparison of examples of multiword units from the CCD, final criteria will be suggested by means of which past participles can be determined to be true adjectives, and thus marked as independent dictionary entries. According to these criteria, the past participles are involved in CCD in two ways: (1) as lenicalised adjectival entries (mljeven - "minced," onečišćen - "polluted") if they show true adjectival 
features within a multiword unit, or (2) under an infinitive verbal entry if they express the passive predicative form (srce je slomljeno - "heart is broken").

\section{The Structure of Multiword Units with Past Participles}

According to attestations in the CCD, past participles appear in multiword units of various syntagmatic forms. Depending on the unit in which they appear, some past participles have both verbal and nominal features, and thus they fall under the homonymic model provided by ZNIKA (2005: 437). It is possible to single out five structures. The first one (presented in section 2.1) includes prototypical participle-noun structures in which the past participles show features of true adjectives. In the other four structures (presented in sections $2.2,2.3,2.4$, and 2.5), the past participles retain verb features expressing the passive voice.

\subsection{Past Participle Form + Noun}

The past participle form + noun structure here is considered the prototypical structure in which the past participle is realised as an attribute agreeing with the head noun in gender, number, and case. According to attestations in the CCD, we can outline the following past participles realised in this type of a multiword structure:

Table 1

Past participles in MWUs in Croatian

\begin{tabular}{|c|c|c|}
\hline No. & Past participles & MWUs with past participles from the CCD \\
\hline 1 & blokiran & $\begin{array}{l}\text { blokirani računi - "blocked accounts" } \\
\text { blokirane prometnice - "blocked highways" }\end{array}$ \\
\hline 2 & donesen & $\begin{array}{l}\text { donesen zakon - lit. "brought," Engl. "enacted law" } \\
\text { donesena odluka / presuda - lit. "brought," Engl. "enacted decision / } \\
\text { judgment" }\end{array}$ \\
\hline 3 & ispečen & $\begin{array}{l}\text { ispečen odrezak - "baked steak" } \\
\text { ispečeno meso - "baked meat" }\end{array}$ \\
\hline 4 & isplaćen & $\begin{array}{l}\text { isplaćen iznos - "paid amount" } \\
\text { isplaćeni troškovi - "paid enpenses" }\end{array}$ \\
\hline 5 & istucan & istucan kamen - "crushed stone" \\
\hline 6 & izražen & izražene razlike - "expressed differences" \\
\hline 7 & izveden & $\begin{array}{l}\text { izvedene mjerne jedinice - "derived units of measurement" } \\
\text { izvedeni broj - "derived number" }\end{array}$ \\
\hline 8 & mljeven & $\begin{array}{l}\text { mljevena kava - "minced coffee" } \\
\text { mljeveni papar - "minced pepper" } \\
\text { mljeveno meso - "minced meat" }\end{array}$ \\
\hline 9 & nacrtan & nacrtane obrue - "drawn eyebrows" \\
\hline 10 & nagrađen & nagrađeni umjetnik / glumac ${ }^{\text {a) }}$ - "awarded artist / actor" \\
\hline 11 & natkriven & $\begin{array}{l}\text { natkriven plivački bazen / stadion - "covered swimming pool / stadium" } \\
\text { natkrivena terasa - "covered terrace" } \\
\text { natkriveno stajalište - "covered station" }\end{array}$ \\
\hline 12 & obilježen & obilježen red riječi / pješački prijelaz - "marked word order / crosswalk" \\
\hline
\end{tabular}


Table 1 continued

\begin{tabular}{|c|c|c|}
\hline 13 & obnouljen & $\begin{array}{l}\text { obnouljena cesta - "renovated road" } \\
\text { obnouljena crkva - "renovated church" } \\
\text { obnouljena obiteljska kuća - "renovated family house" } \\
\text { obnouljena pročelja - "renouated facades" }\end{array}$ \\
\hline 14 & ocijenjen & $\begin{array}{l}\text { ocijenjeni pisani radovi - "evaluated written works" } \\
\text { ocijenjeni proizvodi - "rated products" }\end{array}$ \\
\hline 15 & očišćen & $\begin{array}{l}\text { očišćene ulice - "cleaned streets" } \\
\text { očišćen put - "cleaned path" }\end{array}$ \\
\hline 16 & odbijen & $\begin{array}{l}\text { odbijen zahtjev - "rejected request" } \\
\text { odbijena molba - "rejected application" }\end{array}$ \\
\hline 17 & odigran & $\begin{array}{l}\text { odigrana utakmica - "played match" } \\
\text { loše odigran servis - "bad played service" }\end{array}$ \\
\hline 18 & odležan & odležano vino - lit. "laid," Engl. "aged wine" \\
\hline 19 & odobren & $\begin{array}{l}\text { odobren iznos - "approved amount" } \\
\text { odobrena cijena - "approved price" }\end{array}$ \\
\hline 20 & ojačan & $\begin{array}{l}\text { ojačan beton - "reinforced concrete" } \\
\text { ojačana konstrukcija / plastika - "reinforced construction / plastic" }\end{array}$ \\
\hline 21 & okovan & $\begin{array}{l}\text { Okovani Prometej - "Prometheus Bound" } \\
\text { okovane noge / ruke - "bound / chained legs / arms" }\end{array}$ \\
\hline 22 & onečišćen & onečišćen potok / zrak - "polluted stream / air" \\
\hline 23 & opkoljen & $\begin{array}{l}\text { opkoljeno selo - "surrounded village" } \\
\text { opkoljena vojska - "surrounded army" }\end{array}$ \\
\hline 24 & otegnut & $\begin{array}{l}\text { otegnut način govora / izgouor - "drawled way of speaking / pronun- } \\
\text { ciation" }\end{array}$ \\
\hline 25 & plaćen & $\begin{array}{l}\text { plaćeni dopust - "paid holiday" } \\
\text { plaćeni računi - "paid invoices" }\end{array}$ \\
\hline 26 & počinjen & $\begin{array}{l}\text { počinjeni zločini - "committed crimes" } \\
\text { počinjeno kazneno djelo - "committed criminal offense" }\end{array}$ \\
\hline 27 & podmetnut & $\begin{array}{l}\text { podmetnut požar / dokaz - "planted fire / evidence" } \\
\text { podmetnuta bomba - "planted bomb" }\end{array}$ \\
\hline 28 & podstavljen & $\begin{array}{l}\text { podstavljen kaput - "padded coat" } \\
\text { podstavljena jakna - "padded jacket" } \\
\text { podstavljeno odijelo - "padded suit" }\end{array}$ \\
\hline 29 & pomiješan & pomiješani osjećaji / okusi - "mined feelings / flavours" \\
\hline 30 & prekinut & $\begin{array}{l}\text { prekinuta veza - "broken connection" } \\
\text { Muzej prekinutih veza }{ }^{\text {b) }} \text { - "Museum of Broken Relationships" } \\
\text { prekinuti luk - "broken arch" }\end{array}$ \\
\hline 31 & preplaćen & $\begin{array}{l}\text { preplaćena filmska zuijezda - "overpaid movie star" } \\
\text { preplaćen igrač / iznos - "overpaid player / amount" }\end{array}$ \\
\hline 32 & presađen & presađena kosa / koža - "transplanted hair / skin” \\
\hline 33 & prijeđen & $\begin{array}{l}\text { prijeđena kilometraža - "passed mileage" } \\
\text { prijeđen put - "passed path" }\end{array}$ \\
\hline 34 & prikupljen & $\begin{array}{l}\text { prikupljen novac - "collected money" } \\
\text { prikupljena sredstva - "collected funds" }\end{array}$ \\
\hline 35 & priložen & $\begin{array}{l}\text { priložena dokumentacija - "enclosed documentation" } \\
\text { priložen dopis - "enclosed letter" }\end{array}$ \\
\hline 36 & ranjen & $\begin{array}{l}\text { ranjena duša - "wounded soul" } \\
\text { ranjeno srce - "wounded heart" } \\
\text { ranjeni civili / vojnici - "wounded civilians / soldiers" }\end{array}$ \\
\hline 37 & sklopljen & sklopljena elektroda - lit. "assembled," Engl. "combination electrode" \\
\hline
\end{tabular}


Table 1 continued

\begin{tabular}{|c|c|c|}
\hline 38 & smanjen & $\begin{array}{l}\text { smanjena vidljivost - "reduced visibility" } \\
\text { smanjena ubrojivost - "reduced accountability" }\end{array}$ \\
\hline 39 & snižen & $\begin{array}{l}\text { snižene cijene - "reduced prices" } \\
\text { sniženi tlak - "reduced pressure" } \\
\text { sniženi šećer - "reduced sugar" }\end{array}$ \\
\hline 40 & spušten & $\begin{array}{l}\text { spušteni strop / krov - "lowered ceiling / roof" } \\
\text { spuštena stopala - "lowered feet" }\end{array}$ \\
\hline 41 & srušen & $\begin{array}{l}\text { srušena stabla - "ruined trees" } \\
\text { srušene kuće - "ruined houses" } \\
\text { srušeni ideali - "ruined ideals" }\end{array}$ \\
\hline 42 & stečen & $\begin{array}{l}\text { stečena imovina - "acquired property" } \\
\text { stečena prava - "acquired rights" } \\
\text { stečeno iskustvo - "acquired experience" }\end{array}$ \\
\hline 43 & ugažen & $\begin{array}{l}\text { ugažen snijeg - "trodden snow" } \\
\text { ugažena staza - "trodden path" }\end{array}$ \\
\hline 44 & uhićen & $\begin{array}{l}\text { uhićeni šverceri / teroristi / prijestupnici - "arrested smugglers / terrorists } \\
\text { / offenders" }\end{array}$ \\
\hline 45 & ukraden & $\begin{array}{l}\text { ukradena roba - "stolen goods" } \\
\text { ukradene umjetnine - "stolen goods" }\end{array}$ \\
\hline 46 & umetnut & $\begin{array}{l}\text { umetnuta rečenica - "inserted sentence" } \\
\text { umetnut redak - "inserted row" }\end{array}$ \\
\hline 47 & usječen & $\begin{array}{l}\text { usječen kanjon - "incised canyon" } \\
\text { usječena brazda - "incised furrow" }\end{array}$ \\
\hline 48 & utrošen & $\begin{array}{l}\text { utrošena sredstua - "spent funds" } \\
\text { utrošen materijal - "spent material" }\end{array}$ \\
\hline 49 & vezen & $\begin{array}{l}\text { vezena marama - "embroidered scarf" } \\
\text { vezen stolnjak - "embroidered tablecloth" }\end{array}$ \\
\hline 50 & vraćen & $\begin{array}{l}\text { vraćen novac - "returned money" } \\
\text { vraćena roba - "returned goods" } \\
\text { vraćeno povjerenje - "returned confidence" }\end{array}$ \\
\hline 51 & zacrtan & zacrtan cilj / plan - "outlined goal / plan" \\
\hline 52 & začuđen & $\begin{array}{l}\text { Začuđeni suatovic) - "The Astonished Wedding Guests" } \\
\text { začuđen pogled - "astonished look" }\end{array}$ \\
\hline 53 & zagarantiran & $\begin{array}{l}\text { zagarantiran uspjeh - "guaranteed success" } \\
\text { zagarantirano pravo - "guaranteed right" }\end{array}$ \\
\hline 54 & zaglauljen & $\begin{array}{l}\text { zaglauljen metak - "stuck bullet" } \\
\text { zaglavljena papučica gasa - "stuck accelerator pedal" }\end{array}$ \\
\hline 55 & zagrađen & $\begin{array}{l}\text { zagrađen prolaz - "gated passage" } \\
\text { zagrađena terasa - "gated terrace" }\end{array}$ \\
\hline 56 & zakazan & $\begin{array}{l}\text { zakazan sastanak - "scheduled meeting" } \\
\text { zakazana sjednica - "scheduled session" }\end{array}$ \\
\hline 57 & zaključen & zaključen slučaj / ugovor - "concluded case / contract" \\
\hline 58 & zakočen & $\begin{array}{l}\text { zakočena tipka - "locked button" } \\
\text { zakočena leđa - lit. "locked," Engl. "stiff back" }\end{array}$ \\
\hline 59 & zakopan & $\begin{array}{l}\text { zakopan talent - "buried talent" } \\
\text { zakopano blago - "buried treasures" }\end{array}$ \\
\hline 60 & zakrčen & $\begin{array}{l}\text { zakrčen prilaz - "blocked access" } \\
\text { zakrčene prometnice - "blocked roads" }\end{array}$ \\
\hline 61 & zaleđen & $\begin{array}{l}\text { zaleđen kolnik - "iced pavement" } \\
\text { zaleđen osmijeh - "iced smile" } \\
\text { zaleđena rijeka - "iced river" }\end{array}$ \\
\hline
\end{tabular}


Table 1 continued

\begin{tabular}{|c|c|c|}
\hline 62 & zamaskiran & zamaskirani pljačkaši / navijači - "masked robbers / fans" \\
\hline 63 & zameten & $\begin{array}{l}\text { zametene ceste - "snowed roads" } \\
\text { zameteni tragovi - "snowed traces" }\end{array}$ \\
\hline 64 & zamrljan & $\begin{array}{l}\text { zamrljan stolnjak - "stained tablecloth" } \\
\text { zamrljane ruke - "stained hands" }\end{array}$ \\
\hline 65 & zamrznut & $\begin{array}{l}\text { zamrznuta hrana - "frozen food" } \\
\text { zamrznut izraz lica / pogled - "frozen face / look" }\end{array}$ \\
\hline 66 & zapaljen & $\begin{array}{l}\text { zapaljene baklje - "ignited torches" } \\
\text { zapaljene strasti - "ignited passions" } \\
\text { zapaljeno sijeno - "ignited hay" }\end{array}$ \\
\hline 67 & zapečaćen & $\begin{array}{l}\text { zapečaćen spis - "sealed file" } \\
\text { zapečaćena omotnica - "sealed envelope" } \\
\text { zapečaćene glasačke kutije - "sealed voting bores" }\end{array}$ \\
\hline 68 & zapisan & $\begin{array}{l}\text { zapisan datum / tekst - "written date / tert" } \\
\text { zapisana riječ Božja - "written word of God" } \\
\text { zapisane pjesme - "written songs" }\end{array}$ \\
\hline 69 & zaplijenjen & $\begin{array}{l}\text { zaplijenjena imovina - "seized assets" } \\
\text { zaplijenjene cigarete - "seized cigarettes" }\end{array}$ \\
\hline 70 & zapriječen & zapriječen ulaz / kolni prilaz - "blocked entrance / access road" \\
\hline 71 & zarađen & $\begin{array}{l}\text { zarađen novac - "earned money" } \\
\text { zarađena plaća - "earned salary" }\end{array}$ \\
\hline 72 & zaražen & $\begin{array}{l}\text { zaražene životinje - "infected animals" } \\
\text { zaraženo meso - "infected meat" }\end{array}$ \\
\hline 73 & zaređen & zaređeni suećenici - "ordained priests" \\
\hline 74 & zasijan & $\begin{array}{l}\text { zasijana pouršina - "seeded surface" } \\
\text { zasijane njive - "seeded fields" }\end{array}$ \\
\hline 75 & zaslađen & $\begin{array}{l}\text { zaslađeno piće - "sweetened drink" } \\
\text { zaslađeni sok - "sweetened juice" }\end{array}$ \\
\hline 76 & zastupan & $\begin{array}{l}\text { zastupana stranka - "represented client" } \\
\text { zastupani interesi - "represented interests" }\end{array}$ \\
\hline 77 & zašiljen & $\begin{array}{l}\text { zašiljena olouka - "sharpened pencil" } \\
\text { zašiljen kolac - "sharpened stake" }\end{array}$ \\
\hline 78 & zatajen & $\begin{array}{l}\text { zatajen podatak - "concealed information" } \\
\text { zatajene nezakonite radnje - "concealed illegal acts" }\end{array}$ \\
\hline 79 & zatočen & zatočeni civili / novinari - "imprisoned civilians / journalists" \\
\hline 80 & zatrauljen & $\begin{array}{l}\text { zatravljena pouršina - "grassed surface" } \\
\text { zatravljen vinograd - "grassed vineyard" }\end{array}$ \\
\hline 81 & zdrobljen & $\begin{array}{l}\text { zdrobljen kamen - "crushed stone" } \\
\text { zdrobljena kreda - "crushed chalk" }\end{array}$ \\
\hline 82 & zgažen & $\begin{array}{l}\text { zgažen narod - "trampled people" } \\
\text { zgaženo cuijeće - "trampled flowers" } \\
\text { zgažen snijeg - "trampled snow" }\end{array}$ \\
\hline 83 & zgotouljen & zgotouljen posao / plan - "realised work / plan" \\
\hline 84 & zgrožen & $\begin{array}{l}\text { zgroženi prolaznici - "horrified passers-by" } \\
\text { zgoržena jaunost - "horrified public" }\end{array}$ \\
\hline
\end{tabular}

a) Punctuation mark / separates the nouns wich are matched in gender, number, and case.

b) The name of museum in Zagreb.

c) The novel of Croatian writer Eugen Kumičić. 
This research includes 84 past participles. All of them systematically fit into the model described for Slavic languages (Smith, 1995: 84; Cetnarowska, 2000; SaAvedra, 2007; KIBORT, 2012); Croatian past participles are mostly related to perfective prefinal verbs ${ }^{6}$ which semantically belong to the class of resultative verbs. Here I agree with the assumption that resultativity is a feature that affects the adjectivisation of passive verb forms, i.e. past participles (Nedjalkov, Jaxontov, 1988; Belaj, 2002; SaAvedra, 2007). The resultativity of participles manifests itself in expressing descriptive meanings or states of the nouns with which past participles co-occur in MWUs. As can be seen from the MWUs in Table 1, there are no semantic limitations as to with which types of nouns past participles are collocable. Generally, nouns that refer to a person, thing, place, or abstract quality can be components of participle-noun structures in Croatian MWUs.

\subsection{Adverb + Past Participle + Noun in Nominative Case}

Multiword structures formed by an adverb, past participle form, and noun can be considered a subgroup of adjective-noun structures, and they are common in MWUs in various fields of use. Syntactically, such multiword units are reduced forms of relative clauses, e.g. usječen - "incised" (perasto usječen list / dlanasto usječen list > list koji je usječen perasto / dlanasto "pinnate / palmate incised leaf > leaf (that is) incised pinnate / palmate"), čuvan - "guarded" (strogo čuvana tajna - "tightly guarded secret," strogo čuvani zatuor - "strictly guarded prison"), ocijenjen - "rated" (negativno ocijenjeni učenici "negatively rated students"), pretučen - "beaten" (teško pretučen mladić - "a badly beaten young man"), utrošen - "spent" (namjenski utrošen novac - "purposefully spent money").

\subsection{Noun + Past Participle + Noun in the Instrumental Case / Prepositional Phrase}

Multiword structures formed by a noun in the nominative case, a past participle, and a noun in the instrumental case or prepositional phrase also can be considered as MWUs with reduced forms of a relative clauses (KAтıčić, 1991: 381), e.g. odrezak ispečen na žaru > odrezak koji je ispečen na žaru - "a steak (that is) grilled on hot coals"; brod okovan ledom > brod koji je okovan ledom - "a ship (that is) trapped in ice"; riblji filet preliven limunovim sokom > riblji filet koji je preliven limunovim sokom - "a fish fillet (that is) drizzled with lemon juice"; grad opkoljen zidinama > grad koji je opkoljen zidinama - "a city (that is) surrounded by walls"; špageti preliveni umakom od rajčica > špageti koji su preliveni umakom od rajčica - "spaghetti (that is) covered with tomato sauce"; urijednost izražena u postotcima > vrijednost koja je izražena u postotcima - "a value (that is) expressed in percent"; zastave spuštene na pola koplja > zastave koje su spuštene na pola koplja - "flags (that are) lowered to half mast." In such a structure of multiword units, the past participles express the passive voice, and, though agreeing with the preceding noun, they do not have features of true adjectives.

${ }^{6}$ Based on the examples from Table 1, these Croatian sufines are distinguished in the formation of perfective verbs: is-/iz-, na-/nat-, o-/ob-/od-/op-, po-/pod-/pre-/pri-, s-, u-, z-/za-. 


\subsection{Noun in the Instrumental Case + Past Participle + Noun}

Multiword structures in which a noun in the instrumental case is followed by a past participle and a noun also have the characteristics of reduced relative clauses. The past participle retains the governing features of the verb it belongs to: zlatom vezen rubac > rubac koji je vezen zlatom - literally "a kerchief (that is) embroidered in gold," čavlima okovan lijes - literally "a casket sealed with nails," snijegom zametene ceste - literally "roads covered with snow," radom postignut uspjeh - literally "success attained through work." This structure, considering the word order, is partially variable with the structure described in 2.3 .

\subsection{Past Participle + Noun in the Instrumental Case / Prepositional Phrase}

In multiword units with past participles and a noun in the instrumental case / prepositional phrase, past participles express the passive voice and they do not have the semantic features of true adjective.

This type of multiword structure is common in idiomatic expressions and multiword units typical of journalistic, administrative, and legislative styles. The past participle is a governing component with verb meaning, which defines the form of the noun components in the multiword units: izražen - "expressed" (izraženo je /što/ u brojkama - "/sth/ is expressed in numbers"), nagrađen (nagrađen je /tko/ pljeskom - "/sb/ was awarded with applause"), ojačan (ojačan željeznim šipkama - "strengthened with iron beams," ojačan je /tko/ vjerom - "/sb/ was strengthened by faith"), rođen (rođen je /tko/ pod sretnom zuijezdom - "/sb/ was born under a lucky star"), zameten (zameten u snijegu - "covered in snow"), zapisan (biti zapisan crnim / zlatnim slovima u/što/ - "to be written in black / gold letters into /sth/"), zaražen (zaražen je /tko/ mržnjom / glistama / nogometom - "/sb/ has been infected with hatred / worms / football"), zaređen (zaređen je /tko/ za biskupa / suećenika / đakona - "/sb/ has been ordained as a bishop / priest / deacon"), zatočen (zatočen je /tko/ protiv suoje volje - "/sb/ has been imprisoned against his / her will," zatočen je /tko/ u vlastitu tijelu - "/sb/ is imprisoned in their own body").

\section{The Stylistic and Usage Features of Past Participles}

Through an analysis of MWUs taken from the CCD in which past participles appear, it is possible to define the stylistic and communicative contert in which such units are used. A search in the corpus hrWaC has shown that these multiword units with past participles are common both in different professional fields (scientific, administrative, legislative) and in journalistic usage, e.g. sklopljena elektroda (in chemistry, literally "assembled electrode," Engl. "combination electrode"), plaćeni dopust (in administration / legislation, "paid holiday"), smanjena ubrojivost (in law, "impaired mental capacity"), mljeveno meso (in butchery and cookery, "minced meat"), prekinuti luk (in architecture and construction, "broken arch"), sniženi šećer (in chemistry and nutrition, literally "reduced sugar / reducing sugar"), onečišćen zrak (in ecology, "polluted air"), presađena koža (in medicine / plastic surgery, "transplanted skin"), teško pretučen mladić / odlikovani sportaši / zgroženi prolaznici 
(in journalism, "a badly beaten young man" / "honoured athletes" / "horrified passers-by / public").

In administrative, legislative, and journalistic practice, the use of past participles has a stylistic purpose, as it replaces larger sentence structures with more concise statements. Such statements are based on brachylogy as a stylistic process or an elliptical manner of expression (BAGIć, 2012) that tends towards truncated predicates that omit copulae (verb biti - "to be"), but which retain their past participle form (SILIĆ, PrAnjković, 2005: 196). The multiword units explored in sections 2.2 to 2.5 are the result of this manner of expression. In light of this, ZNIKA (2005: 436-437) makes the important conclusion that the presence of past participles in the terminology of various professional fields also contributes to the adjectivisation of past participles and their inclusion into nominal words.

\section{Conclusion}

By comparing past participles in MWUs from the CCD which co-occur with different nouns and appear as past participles in Croatian dictionaries, and relying on the aforementioned theoretical observations (Cetnarowska, 2000; Belaj, 2002; ZniKa, 2005; SaAvedra, 2007; SeIfArt, 2010; Kibort, 2012), it is possible to outline four criteria for determining the status of past participles in Croatian dictionaries ${ }^{7}$ both as forms of verbal entries and as lexicalised adjectives. The emphasis is placed on their status as independent lexical (adjectival) units:

- Grammatical features such as gender, number, case, and comparison, apart from the part of the verb paradigm, categorise past participles as words with nominal categories. As nominal words, past participles behave as true adjectives and are collocable with nouns of wide semantic potential (e.g. zakopano blago - "buried treasure," pomiješani osjećaji "mired feelings," smanjena vidljivost - "reduced visibility"). The collocatability is certainly an important criterion in the process of adjectivisation of past participles.

- Adjectivisation of past participles is one of the most common ways of forming multiword terms in Croatian used in different scientific and professional fields. The high frequency of such terms indicates that adjectivisation is not a sporadic phenomenon, but rather a common way of forming terminological units in Croatian. ${ }^{8}$

- In stylistically marked language use, especially in the journalistic and administrative style, short forms based on nominalisation are typical (BELAJ, 2002; SiLıć, 2006: 65-66). Verbal constructions convert to nominal ones, which is especially evident in truncating of verbal predicate. Instead, in passive predicative forms (e.g. zahtjev koji je odbijen - "request which is rejected," dokumentacija koja je priložena - "documentation which is enclosed"), past participles are collocatable with nouns (odbijen zahtjev - "rejected request," priložena dokumentacija - "enlosed documentation"). Adjectivisation of past participles motivated

\footnotetext{
${ }^{7}$ Here we are focused on Croatian, but these criteria are also valid for other Slavic languages.

${ }^{8}$ E.g. in hrWaC the frequency of past participle mljeven ("minced") in adjectival use is much more frequent then in verbal (predicative) use: the term mljeveno meso ("minced meat") occurs 1,360 times while the predicative form je mljeven ("is minced") occurs 21 times. (accessed 14 ${ }^{\text {th }}$ February 2019).
} 
by stylistic needs is by no means a negligible criterion for determining participles as independent lexical units.

- Although I have not relied more on statistical data here, the attestation of past participles in adjectival use in the corpus (see footnote 8) is also an important indicator in determining the degree of adjectivisation of past participles. Good lexicographic practice should not ignore corpus evidence of past participles in multiword units.

I consider these criteria useful also in the selection and inclusion of past participles in Croatian dictionaries as stand-alone adjectives, not only as verbal forms. Modern dictionaries are increasingly published online; as electronic publications, they offer the ability to add new entries when needed, since there is no conceptual limit as compared to printed editions. Publishing on the internet has created the conditions for past participle forms: in accordance with their homonymous nature and depending on their semantic and usage potential, participles can be listed both under verbal entries and as independent adjectival entries.

Corpora, databases, and dictionaries

hrWaC - Croatian web corpus [online: http://nlp.ffzg.hr/resources/corpora/hrwac/; accessed: 10.03.2018].

HJP - Hrvatski jezični portal [online: http://hjp.znanje.hr/; accessed: 22.10.2017].

CCD - Kolokacijska baza hruatskoga jezika (Croatian Collocation Database) [online: http://ihjj.hr/ kolokacije/; accessed: 20.03.2018].

RHJ - ŠonJE J., ed., 2000: Rječnik hruatskoga jezika. Zagreb.

ŠKRJ - BIRTIĆ M. et al., 2012: Školski rječnik hrvatskoga jezika. Zagreb.

VRH - Joıı́ Lj., ed., 2015: Veliki rječnik hrvatskoga standardnog jezika. Zagreb.

\section{References}

BAGIĆ K., 2012: Rječnik stilskih figura. Zagreb.

Belaj B., 2002: Nominalizacija kao strategija pasivizacije. "Suuremena linguistika" No. 28/53-54, pp. 11-13.

Benson M., Benson E., Ilson R., 1997: The BBI Dictionary of English Word Combinations. Amsterdam-Philadelphia.

Blagus Bartolec G., 2014: Riječi i njihovi susjedi: Kolokacijske sueze u hrvatskom jeziku. Zagreb.

Cetnarowska B., 2000: Resultative Adjectives in Polish. "Acta Linguistica Hungarica” No. 47, pp. 4779.

IVIR V., 1992/1993: Kolokacije i leksičko značenje. “Filologija” No. 20/21, pp. 181-189.

KAтıčıć R., 1991: Sintaksa hruatskoga knjižeunog jezika. Zagreb.

Kibort A., 2012: Participles, Adjectives, and the Role of Argument Structure. In: Butt, M., Holloway

KING, T., eds., Proceedings of the LFG2012 Conference. Stanford, CA, pp. 323-340.

Marvin T., 2003: Past Participles in Reduced Relatives: A Cross-linguistic Perspective. "Linguistica" No. 43, pp. 141-160.

Mé'čuк I., 2001 [1998]: Collocations and Lexical Functions. In: Cowie A.P., ed., Phraseology: Theory, Analysis and Applications. Orford-New York, pp. 23-53. 
Nedjalkov V.P., Jaxontov S.J., 1988: The Typology of Resultative Constructions. In: Nedjalkov, V.P., ed., Typology of Resultative Constructions. Amsterdam, pp. 3-62.

Ramisch C., 2015: Multiword Expressions Acquisition: A Generic and Open Framework. Basel.

Sag I., Baldwin T., Bond F., Copestake A., Flickinger D., 2002: Multiword Eupressions: A Pain in the Neck for NLP. "Lecture Notes in Computer Science" No. 2276, pp. 1-15.

SaAvedra D., 2007: Pasiune konstrukcije u hruatskome i bugarskome jeziku. “Lahor" No. 4, pp. 217241.

Seifart F., 2010: Nominal Classification. “Language and Linguistics Compass” No. 4 (8), pp. 719-736.

SiLıĆ J., 2006: Funkcionalni stilovi hruatskoga standardnog jezika. Zagreb.

Silić J., Pranjković I., 2005: Gramatika hruatskoga jezika za gimnazije i visoka učilišta. Zagreb.

Sмıтн, N.S., 1995: The Treatment of Participles in Nudožerský's Grammaticae Bohemicae..., Blahosau's Grammatika češka and Rosa's Čechořečnost. “Sborník prací filozofické fakulty brněnské univerzity Studia minora facultatis philosophicae universitatis Brunensis" No. A43, pp. 81-91.

ZNıKA M., 2005: Status glagolskih pridjeva. “Rasprave: Časopis Instituta za hruatski jezik i jezikosloulje" No. 31, pp. 429-440.

Goranka Blagus Bartolec

Past Participles in Multiword Units in Croatian

Summary

Past participles (ending with - $n(-a n,-e n)$, and $-t$ ) and the group of adjectives referred to in Croatian linguistics as true adjectives are similar regarding their syntactic structure. The paper analyses the collocation potential of the past participles based on data from the Croatian Collocation Database (http://ihjj.hr/kolokacije/english/) developed at the Institute of Croatian Language and Linguistics. The article describes both the use of the past participles in the multiword units in Croatian and their status in contemporary Croatian dictionaries. What is described are the criteria for distinguishing the past participles as morphological forms of the verb headword from the lexicalised adjectives that should have the status of independent headwords in the dictionary. 